\title{
(⿸丆口
}

\section{Uma visão geral das manifestações orais de doenças gastrointestinais.}

Ana Helena Junqueira (1)

\section{REVISÃO DE LITERATURA}

Resumo

A cavidade oral faz parte do sistema gastrointestinal e, como tal, a presença de alterações nesta região pode ser o primeiro sinal de doenças sistémicas e gastrointestinais. Como essas alterações são muito comuns, principalmente em crianças, é importante que o dentista saiba quando elas são expressão de um quadro gastrointestinal. O objetivo desta revisão é fornecer ao dentista dados úteis para o diagnóstico, tratamento e manejo das condições mais comuns, como doença de Crohn, colite ulcerativa, doença do refluxo gastroesofágico e doença celíaca. Uma das alterações orais mais comuns é a erosão dentária, com perda de esmalte e aumento do risco de cárie dentária, que tem sido relatada em crianças e adolescentes com refluxo gastroesofágico. Hipoplasia do esmalte dentário e úlceras aftosas são mais comuns em crianças com doença celíaca do que na população em geral. Outra alteração oral muito comum é a gengivite, que afeta 9-95\% das crianças na Europa e na América do Norte e mais de $60 \%$ dos adolescentes. Pioestomatite vegetante pode ser um sinal de colite ulcerosa e doença de Crohn, esta última também foi relacionada a edema difuso da mucosa, mucosa de paralelepípedo, muco-gengivite localizada, ulceração linear profunda, marcas de tecido fibroso, pólipos, nódulos e úlceras aftosas. O reconhecimento imediato de doenças sistêmicas e gastrointestinais por meio de um exame cuidadoso da cavidade oral pode ser o primeiro passo para novas investigações que podem levar a um diagnóstico precoce e tratamento oportuno.

Palavras-chave: Alterações da cavidade oral, Doença celíaca, Doença de Crohn, Doença do refluxo gastroesofágico, Doenças gastrointestinais, Colite ulcerativa. 


\title{
An overview of the oral manifestations of gastrointestinal diseases.
}

\begin{abstract}
The oral cavity is part of the gastrointestinal system and, as such, the presence of changes in this region can be the first sign of systemic and gastrointestinal diseases. As these changes are very common, especially in children, it is important for the dentist to know when they are an expression of a gastrointestinal condition. The objective of this review is to provide the dentist with useful data for the diagnosis, treatment and management of the most common conditions, such as Crohn's disease, ulcerative colitis, gastroesophageal reflux disease and celiac disease. One of the most common oral changes is dental erosion, with loss of enamel and increased risk of dental caries, which has been reported in children and adolescents with gastroesophageal reflux. Dental enamel hypoplasia and aphthous ulcers are more common in children with celiac disease than in the general population. Another very common oral disorder is gingivitis, which affects $9-95 \%$ of children in Europe and North America and more than 60\% of adolescents. Pyostomatitis vegetans can be a sign of ulcerative colitis and Crohn's disease, the latter has also been related to diffuse mucosal edema, cobblestone mucosa, localized mucus-gingivitis, deep linear ulceration, fibrous tissue marks, polyps, nodules and aphthous ulcers. The immediate recognition of systemic and gastrointestinal diseases through a careful examination of the oral cavity may be the first step towards further investigations that can lead to an early diagnosis and timely treatment.
\end{abstract}

Keywords: Disorders of the oral cavity, Celiac disease, Crohn's disease, Gastroesophageal reflux disease, Gastrointestinal disorders, Ulcerative colitis.

Instituição afiliada: 1- Professora da graduação em Odontologia da Faculdade de Palmas, Palmas Tocantins- Brasil.

Dados da publicação: Artigo recebido em 03 de julho, revisado em 11 de julho, aceito para publicação em 20 de julho e publicado em 30 de julho.

DOI: https://doi.org/10.36557/2674-8169.2021v3n7p11-25

Ana Helena Junqueira ahj67@gmail.com

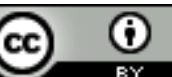

This work is licensed under a Creative Commons Attribution 4.0 International

License. 


\section{INTRODUÇÃO}

Correlações entre alterações na cavidade oral e condições sistêmicas têm sido amplamente relatadas (Chi, 2010; Majorana, 2010) e durante o exame bucal também é possível detectar sinais e sintomas de doenças sistêmicas, como mucocutâneas, distúrbios imunológicos, doenças hormonais, condições hematológicas, infecções sistêmicas e problemas nutricionais (US Department of Health, 2000). Em particular, alterações na cavidade oral, por fazer parte do sistema gastrointestinal (GI), podem revelar uma doença $\mathrm{Gl}$, como doença celíaca, doença do refluxo gastroesofágico ou doença inflamatória intestinal.

As manifestações orais incluem defeitos do esmalte dentário, cáries dentárias e úlceras aftosas que foram relatadas como ocorrendo em indivíduos com doença celíaca e regredindo com uma dieta sem glúten [Pastore, 2008]. A erosão dentária pode ser uma consequência da doença do refluxo gastroesofágico [Tolia, 1997; Bishop, 1994; Schroeder, 1995], e o último pode ser diagnosticado em 25-83\% dos pacientes com cárie, muitos dos quais são crianças [-Smith, 2015]. Vale ressaltar que nos últimos anos um aumento da erosão dentária tem sido destacado (Mulic et al., 2013); na Europa, sua prevalência foi relatada como sendo superior a 50\% em adultos jovens (idade 18-35 anos) (Bartlett et al., 2013).

Outras manifestações orais comuns na doença de Crohn incluem língua geográfica, úlceras, estomatite e doença periodontal [Bishop, 1972; Stankler, 1972; Van Dyke, 1986]. Além disso, as alterações orais podem ser detectadas em até um terço dos pacientes pediátricos com colite ulcerosa e geralmente são inespecíficas [Katsanos, 2015].

O espectro das lesões bucais é amplo, principalmente em crianças, e por isso é importante que o dentista entenda quais são as mais comumente associadas às doenças gastrointestinais, bem como sua abordagem e manejo, a fim de fazer um diagnóstico diferencial correto e fornecer o tratamento adequado. . O papel do dentista é muito importante nessas situações, pois os sintomas muitas vezes não são reconhecidos ou esquecidos pelos gastroenterologistas (Harty et al., 2005) e em muitos casos o diagnóstico é feito quando um especialista em medicina oral ou odontologia está envolvido (Pittock et al. ., 2001; Campbell et al., 2011; Lankarani et al., 2013).

O objetivo deste artigo é revisar a literatura sobre lesões bucais associadas a doenças gastrointestinais, a fim de fornecer dados úteis para o diagnóstico, tratamento e manejo das doenças mais comuns, como doença de Crohn, colite ulcerativa, doença do refluxo gastroesofágico. e doença celíaca.

\section{Doença de crohn}

Desde meados da década de 1970, a incidência da doença de Crohn (CrD), uma condição inflamatória recorrente crônica, aumentou nos países ocidentais 
( Hovde e Moum , 2012), com uma prevalência variando de menos de 10 a cerca de 150 por 100.000 habitantes nos países europeus (Yapp et al., 2000; Gheorghe et al., 2004). A CrD tem uma etiologia complexa e sua patogênese ainda é incerta (Crippa et al. 2016).

Lesões na cavidade oral podem representar uma manifestação inicial e primária da CrD [Daley, 2007; Hussey, 2011; Boirivant, 2012] ou pode ocorrer simultaneamente, ou após o início do envolvimento GI [Kalmar, 2000]; no entanto, lesões orais sem envolvimento gastrointestinal são raramente relatadas na literatura [Zbar, 2012; Fatahzadeh, 2009; Chi, 2010; Daley, 2007]. Essas lesões podem ser uma consequência de baixos níveis séricos de micronutrientes e macronutrientes secundários à má absorção [Jacobs, 1968] ou a reações imunológicas locais a antígenos orais típicos da CrD [Lehner, 1972; Basu, 1976].

A DRO oral pode ser detectada na mucosa bucal, lábios, língua, palato duro e mole, glândulas salivares, gengiva e dentes com alterações que podem ser típicas e patognomônicas, na maioria dos casos associadas ou altamente suspeitas de doença inflamatória intestinal, ou inespecíficas [Katsanos, 2015].

A prevalência de Oral CrD é de cerca de $20 \%$, mas foi relatada até $50 \%$ (Laube et al, 2017; Pittock et al., 2001; Rowland et al., 2010; Lankarani et al., 2013), especialmente em as faixas etárias mais jovens, visto que esses sujeitos parecem ter uma idade de manifestação menor em relação à população média de DCE (Mahid et al., 2008).

In up to $5-15 \%$ of subjects affected by CrD a typical acute oral manifestation, defined orofacial CrD, may arise and show as recurrent or persistent lip swelling, cobblestone of the oral mucosa, stomatitis, mucogingivitis, deep linear or serpiginous ulcerations surrounded by epithelial hyperplasia, tissue tags or polyps, often related to Candida-associated angular cheilitis [Boirivant, 2012; Fatahzadeh, 2009; Katsanos, 2015; Tilakaratne, 2008; Kolho, 2011; Wiesenfeld, 1985; Van der Waal, 2002]. Macroscopic and histological characteristics of this manifestation are similar to those found in the gastrointestinal tract [Stankler, 1972] and can be associated with pain on touch or on eating acidic or spicy foods, impairment of oral function, eating, speaking, and psychosocial stress [Plauth, 1991]. Characteristic oral alterations of CrD include orofacial $\mathrm{CrD}$, granulomatous cheilitis and pyostomatitis vegetans.

O diagnóstico pode ser muito difícil, uma vez que a CrD orofacial é indistinguível da granulomatose orofacial, que pode ser detectada em várias condições (como sarcoidose, queilite granulomatosa de Miescher, síndrome de Melkersson-Rosenthal, granuloma de corpo estranho, rosácea e várias doenças infecciosas granulomatosas) [Wiesenfeld , 1985; Bogenrieder, 2003]. Até 40-50\% dos pacientes jovens com granulomatose orofacial podem desenvolver $\mathrm{CrD}$ e ela pode ser notada mesmo anos após o primeiro aparecimento dos sintomas orais [Rowland, 2010].

Harty et al. [2005] relataram que a inflamação granulomatosa estava presente em $100 \%$ das biópsias coletadas em seu estudo, ressaltando que a mucosa oral representa um local de fácil acesso para a coleta de material diagnóstico.

A queilite granulomatosa é uma inflamação granulomatosa incomum subaguda envolvendo a área dos lábios [Allen, 1990] e é descrita como um inchaço súbito inicial 
dos lábios, principalmente o inferior, que remite em horas ou dias, mas é seguido por edema permanente e inchaço irregular [Friedrich, 1990; Alawi, 2005]. Esta condição também pode ser um sinal de outras doenças, como alergia, sarcoidose, síndrome de Melkersson-Rosenthal, herpes simples recorrente, erisipela recorrente, câncer e doenças genéticas [Katsanos, 2015].

Pioestomatite vegetante é uma manifestação rara que mostra mucosa oral espessada e eritematosa coberta por pústulas e erosões superficiais com padrão de "pegadas de caracol". Tem sido associada em $75 \%$ dos casos com doença inflamatória intestinal [Ayangco, 2002; Lankarani, 2013; Delaporte, 1998] e também pode ser uma expressão de doenças e infecções penfigóides autoimunes [Hansen, 1983].

Outras lesões orais sugestivas de CrD são paralelepípedo, mucogengivite, hipertrofia gengival, edema labial com fissuras verticais, fissura labial na linha média, úlceras lineares profundas da mucosa bucal e labial e lesões endurecidas semelhantes a marcadores [Greenstein, 1976; Lisciandrano, 1996; Field, 1989; Colella, 1971; Lourenço, 2010].

A DRC também foi relacionada a lesões inespecíficas, como estomatite aftosa recorrente (RAS), boca seca, fístula do ducto salivar, infecções bucais recorrentes, espaço bucal persistente, abscessos assépticos, ulcerações pustulares, eritema, inchaço e pavimentação da gengiva, mandibular osteomielite [Correl, 1981; Delaporte, 1998; Gargiulo, 1989; Ciantar, 2007].

Quanto aos exames diagnósticos, Van der Waal et al. [2002] não recomendam investigação de rotina do trato gastrointestinal em pacientes com história negativa de doenças gastrointestinais, enquanto Plauth et al. [1991] recomendam investigações exaustivas e repetidas para $\mathrm{CrD}$ em casos de granulomatose orofacial, mesmo quando nenhum sintoma gastrointestinal está presente; a endoscopia é recomendada em pacientes com sintomas intestinais ou / e tipos altamente específicos de lesões orais [Katsanos, 2015]. A triagem periódica da calprotectina fecal, um marcador substituto para a inflamação da mucosa (por exemplo, na $\mathrm{CrD}$ ), também é sugerida [Roseth, 1999; Sipponen, 2008].

O tratamento da CrD oral pode incluir terapia com esteróides [Hussey, 2011; Harikishan, 2012], agente antagonista do fator de necrose tumoral alfa infliximabe (administrado com resultados promissores em queilite granulomatosa crônica e em um caso de CrD orofacial e inchaço labial não responsivo a qualquer outro tratamento) [Peitsch, 2007; Barry, 2005] e, raramente, metotrexato.

\section{Colite ulcerativa}

A colite ulcerativa (UC) é considerada com DCC o tipo mais comum de doença inflamatória intestinal, com maior incidência em indivíduos com idade entre 15 e 25 anos e um segundo, menor pico entre 55 e 65 anos (Mantegazza et al., 2016a).

Assim como a CrD, também a UC tem base imunológica [Lourenço, 2010), a UC e a CrD também compartilham algumas manifestações clínicas, como úlceras mucosas ( Seo, 1992) ), piomatite vegetante (Alstead , 1991; Calobrisi, 1995), pústulas difusas (O

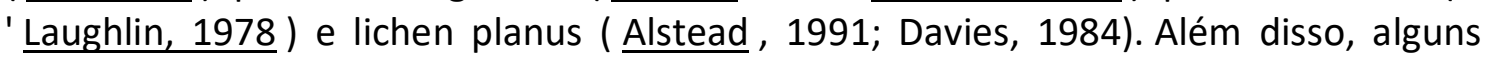


medicamentos usados para o tratamento de doenças gastrointestinais às vezes são responsáveis por efeitos colaterais orais (Parvinen, 1984; Sreebny, 1986).

Novamente, a pioestomatite vegetante se manifesta como múltiplas pústulas miliares brancas ou amarelas que podem se fundir em úlceras em trilha de caracol, com uma base mucosa eritematosa e edematosa envolvendo principalmente a gengiva labial e as mucosas labial e bucal. Pode ser diagnosticado quando uma combinação de características clínicas de doença inflamatória intestinal, eosinofilia periférica, achados histológicos e cultura negativa do exsudato estão presentes [Mijandrusi-Sinci, 2010]. A idade média no diagnóstico geralmente é de 34 anos.

Outras manifestações orais inespecíficas na CU são aftas orais (5-10\% dos pacientes), glossite, queilite, estomatite, líquen plano, úlceras mucosas, pústulas difusas e gengivite inespecífica [Folashade, 2008; Lekovi, 2011; Krebs, 2011].

O diagnóstico de CU é baseado na presença de diarreia com sangue com culturas de fezes negativas e inflamação difusa da mucosa contínua envolvendo o reto e se estendendo até um ponto mais proximal no cólon na avaliação endoscópica [Kugathasan, 2003; Bentsen, 2002].

O tratamento inclui esteróides orais [Thrash, 2013]. No entanto, uma avaliação odontológica pode ser útil durante a investigação de pacientes com suspeita de CU (Elahi, 2012).

\section{Doença do refluxo gastroesofágico}

O refluxo gastroesofágico é o movimento fisiológico do conteúdo gástrico para o esôfago e orofaringe que ocorre através do relaxamento do esfíncter esofágico inferior; pode se tornar patológica [Colletti, 2003] quando ocorrem regurgitações repetidas, náuseas, azia, tosse, laringite, asma ou pneumonia e, portanto, é definida como doença do refluxo gastroesofágico (DRGE). Também pode ser acompanhada por complicações secundárias, como esofagite, hemorragia, estenose, esôfago de Barrett e adenocarcinoma [Vakil, 2006].

Uma forte associação entre GERD e DE foi relatada ( Pace , 2008). As diretrizes da NASPGHAN e ESPGHAN de 2009 sobre refluxo em crianças relatam que as manifestações orais também foram descritas na DRGE e, em particular, sua correlação com erosões dentárias [Vandenplas, 2009; Pindborg, 1970]; de fato, sua prevalência em crianças com DRGE foi relatada em 83,3\% [Dahshan et al., 2010], embora na literatura sejam relatados números diferentes.

Vale ressaltar que em indivíduos com DRGE a erosão dentária envolve principalmente a dentição mista e começa na área posterior, em particular nas superfícies facial, oclusal e lingual [Dahshan et al., 2010]. Nessas situações, a administração de inibidores da bomba de prótons tem se mostrado eficaz [Wilder-Smith et al., 2009]. 
A DRGE também pode ser a causa de alterações nos tecidos moles e no fluxo salivar [Silva, 2001]; Di Fede et al. [2008] relataram uma associação significativa de DRGE com sensação de queimação ácida oral, xerostomia, halitose subjetiva e palato duro mole e eritema da mucosa da úvula.

\section{Doença celíaca}

A doença celíaca (DC) é uma condição autoimune que pode se desenvolver como consequência do consumo de glúten [Barker e Liu, 2008] em indivíduos geneticamente suscetíveis. Sua incidência aumentou nas últimas décadas e atualmente é um dos distúrbios mais comuns ao longo da vida em todo o mundo (Mantegazza et al. 2016b), com uma prevalência média estimada de 0,9\% [Lionetti, 2014].

As alterações orais relacionadas à DC incluem: defeitos do esmalte dentário, erupção tardia, estomatite aftosa recorrente, glossite atrófica, xerostomia, queilose e líquen plano oral [Rashid, 2011; Tack, 2010].

Como os defeitos do esmalte dentário são mais comuns em pacientes com DC do que na população em geral [Rashid, 2011], a presença de defeitos específicos do esmalte dentário é considerada sugestiva da doença [Hill, 2005], especialmente quando aparecem simétrica e cronologicamente em todos 4 quadrantes, com mais defeitos nos incisivos e molares superiores e inferiores que frequentemente apresentam cúspides intactas [Rashid, 2011]. Uma classificação dos defeitos localizados do esmalte em DC foi desenvolvida por Aine et al. em 1990, incluindo corrosão, sulcos e, às vezes, perda total do esmalte.

Campisi et al. [2008] e Condò et al. (2011) observaram uma maior ocorrência de erupção retardada em crianças com DC em comparação com crianças saudáveis.

Outra condição muito comum na DC é a estomatite aftosa recorrente. Essa doença ulcerativa oral afeta de $10 \%$ a $20 \%$ da população geral e, de acordo com Sedghizadeh et al. (2002), 41\% dos indivíduos com DC têm história dessa alteração oral em comparação com $27 \%$ dos controles saudáveis. Manifesta-se com úlceras únicas ou múltiplas recorrentes na mucosa oral, de formato redondo ou ovoide, com halo eritematoso e assoalho amarelo ou cinza.

Glossite atrófica é uma condição caracterizada por língua vermelha, lisa e brilhante, causada por deficiência de vitamina B12 [Trandafir, 2014]; xerostomia devido à disfunção da glândula salivar com fluxo salivar diminuído que se desenvolve após a exposição ao glúten [Lähteenoja, 1998; Shetyer, 2013; Ertekin, 2005]; queilite angular devido à má absorção [Rashid, 2011].

Até 2012, a positividade para anticorpos anti-transglutaminase e / ou antiendomísio associados a achados histológicos característicos com base em múltiplas biópsias do duodeno eram necessários para diagnosticar DC [Benkebil, 2013]. Atualmente, um algoritmo que pode ser encontrado nas diretrizes pediátricas europeias permite evitar a biópsia em crianças com sintomas clínicos típicos, título de anticorpo anti-transglutaminase $>10$ vezes com anticorpos anti-endomísio positivos e um genótipo compatível com o desenvolvimento de CD [Husby, 2012] . 
Em todos os casos, uma dieta sem glúten é o tratamento mais eficaz para DC [Husby, 2012].

\section{CONCLUSÃO}

Considerando a alta frequência de alterações que podem surgir na cavidade oral das crianças, o dentista deve ser capaz de detectar aquelas lesões que necessitam de uma investigação diagnóstica minuciosa. Nestes casos, uma abordagem multidisciplinar, incluindo gastroenterologistas, radiologistas e cirurgiões, bem como dentistas e especialistas em medicina oral, poderia melhorar o diagnóstico e a gestão do paciente (Laube et al., 2018).

Lesões da mucosa oral, especialmente em crianças, são pistas importantes para diagnosticar doenças sistêmicas das quais podem ser o sinal de apresentação primário, precedendo os sintomas gastrointestinais. Uma das primeiras etapas para o diagnóstico de doenças gastrointestinais começa com um exame clínico odontológico de toda a cavidade oral e uma história médica detalhada (Mantegazza et al., 2016c); portanto, os dentistas são incentivados a inquirir sobre os sintomas associados a esses distúrbios e a história familiar de doenças gastrointestinais que representam potenciais fatores etiológicos associados às doenças bucais. Como a presença de alterações bucais suspeitas requer cooperação interdisciplinar, a comunicação entre gastroenterologistas e dentistas é imprescindível para o sucesso do tratamento.

\section{THE AUTHORS DECLARE NO CONFLICTS OF INTEREST.}

\section{REFERÊNCIAS}

1. Italian Ministry of Health, Guidelines. Linee guida nazionali per la promozione della salute orale e la prevenzione delle patologie orali in età evolutiva, 2014 Ministero del lavoro, della Salute e delle Politiche Sociali. 2013.

2. Chi AC, Neville BW, Krayer JW, Gonsalves WC. Oral manifestations of systemic disease. Am Fam Physician 2010 Dec 1;82(11):1381-8.

3. Majorana A, Bardellini E, Flocchini P, Amadori F, Conti G, Campus G. Oral mucosal lesions in children from 0 to 12 years old: ten years' experience. Oral Surg Oral Med Oral Pathol Oral Radiol Endod 2010 Jul;110(1):e13-8. doi: 10.1016/j.tripleo.2010.02.025. Epub 2010 May 10.

4. US Department of Health and Human Services. Oral Health in America: A Report of the Surgeon General. Rockville, MD: US Department of Health and Human Services, National Institute of Dental and Craniofacial Research, National Institutes of Health: 2000; 1-308.

5. Pastore L, Campisi G, Compilato D, Lo Muzio L. Orally based diagnosis of celiac disease: current perspectives. J Dent Res 2008; 87(12): 1100-7. 
6. Pastore L, Carroccio A, Compilato D, Panzarella V, Serpico R, Lo Muzio L. Oral manifestations of celiac disease. J Clin Gastroenterol 2008;42(3):224-32.

7. Tolia V. Evaluation and management of pediatric gastroesophageal reflux. Fam Pract Recert 1997;19:35-57.

8. Bishop RP, Brewster AC, Antonioli DA. Crohn's disease of the mouth. Gastroenterology 1972;62:302-6.

9. Bishop K, Briggs $P$, Kelleher $M$. The aetiology and management of localized anterior tooth wear in the young adult [review]. Dent Update 1994;21:153-60.

10. Schroeder P, Filler S, Ramirez B, Lazarchik D, Vaezi M, Richter J. Dental erosion and acid reflux disease. Ann Intern Med 1995;122:809-15.

11. Smith CH1, Materna A2, Martig L3, Lussi A4. Gastro-oesophageal reflux is common in oligosymptomatic patients with dental erosion: $\mathrm{A} \mathrm{pH}$-impedance and endoscopic study. United European Gastroenterol J 2015 Apr;3(2):174-81.

12. Mulic $A$, Tveit $A B$, Skaare $A B$. Prevalence and severity of dental erosive wear among a group of Norwegian 18-year-olds. Acta Odontol Scand 2013;71:475-481.

13. Bartlett DW, Lussi A, West NX, Bouchard P, Sanz M, Bourgeois D. Prevalence of tooth wear on buccal and lingual surfaces and possible risk factors in young European adults. J Dent 2013;41:1007-1013.

14. Stankler L, Ewen SWB, Kerr NW. Crohn's disease of the mouth. Br J Dermatol 1972;87:5014.

15. Van Dyke TE, Dowell VR Jr, Offenbacher S, Snyder W, Hersh T. Potential role of microorganisms isolated from periodontal lesions in the pathogenesis of inflammatory bowel disease. Infect Immun 1986;53:671-7.

16. Katsanos KH, Torres J, Roda G, Brygo A, Delaporte E, Colombel JF. Review article: nonmalignant oral manifestations in inflammatory bowel diseases. Aliment Pharmacol Ther 2015 Jul; 42(1): 40-60.

17. Harty $S$, Fleming $P$, Rowland $M$ et al. A prospective study of the oral manifestations of Crohn's disease. Clinical Gastroenterology Hepatology 2005; 3 (9): 886-891.

18. > Pittock S, Drumm B, Fleming P et al.The oral cavity in Crohn's disease. J. Pediatr 2001; 138: 767-771.

19. Campbell H, Escudier M, Patel $P$ et al. Distinguishing orofacial granulomatosis from Crohn's disease: two separate disease entities? Inflamm Bowel Dis 2011; 17: 2109-2115.

20. Lankarani KB, Sivandzadeh GR, Hassanpour S. Oral manifestation in inflammatory bowel disease: a review. World J Gastroenterol 2013; 19: 8571-8579.

21. Hovde $\varnothing$, Moum BA. Epidemiology and clinical course of Crohn's disease: Results from observational studies. World J Gastroenterol. 2012 Apr 21; 18(15): 1723-1731.

22. Yapp TR, Stenson R, Thomas GA, Lawrie BW, Williams GT, Hawthorne AB. Crohn's disease incidence in Cardiff from 1930: an update for 1991-1995. Eur J Gastroenterol Hepatol. 2000;12:907-911.

23. Gheorghe C, Pascu O, Gheorghe L, lacob R, Dumitru E, Tantau M, Vadan R, Goldis A, Balan $G$, lacob $S$, et al. Epidemiology of inflammatory bowel disease in adults who refer to gastroenterology care in Romania: a multicentre study. Eur J Gastroenterol Hepatol 2004;16:1153-1159.

24. Crippa R, Zuccotti GV, Mantegazza C. Oral manifestations of gastrointestinal diseases in children. Part 2: Crohn's disease. Eur J Paediatr Dent 2016;17(2):164-166.

25. Daley TD, Armstrong JE. Oral manifestations of gastrointestinal diseases. Can J Gastroenterol 2007; 21: 241-244.

26. Hussey S, Fleming P, Rowland M, Harty S, Chan L, Broderick A et al. Disease outcome for 
children who present with oral manifestation of Crohn's disease. Eur Arch Paediatr Dent 2011; 12:167-9.

27. Boirivant M, Cossu A. Inflammatory bowel disease. Oral Diseases 2012; 18 (1): 1-15.

28. Kalmar JR. Crohn's disease: orofacial considerations and disease pathogenesis. Periodontology 2000 1994; 6: 101-115.

29. Zbar AP, Ben-Horin S, Beer-Gabel M, Eliakim R. Oral Crohn's Disease: is it a separable disease from orofacial granulomatosis? J Crohns Colitis 2012;6:134-42.

30. Fatahzadeh M, Schwartz RA, Kapila R, Rochford C. Orofacial Crohn's disease: an oral enigma. Acta Dermatovenerol Croat 2009;17:289-300.

31. Jacobs A, Cavill I. The oral lesions of iron deficiency anaemia: pyridoxine and riboflavin status. Br J Haematol 1968;14:291-5.

32. Lehner T. Cell-mediated immune response in oral disease: a review. J Oral Pathol 1972;1:39-58.

33. Basu MK. Oral manifestations of Crohn's disease: studies in the pathogenesis. Proc R Soc Med 1976;69:765-6.

34. Mahid SS, Mulhall AM, Gholson RD et al. Inflammatory bowel disease and African Americans: a systematic review. Inflamm Bowel Dis 2008;14: 960-

35. Laube R, Liu K, Schifter M, Yang JL, Suen MK, Leong RW. Oral and upper gastrointestinal Crohn's disease. J Gastroenterol Hepatol 2018 Feb;33(2):355-364.

36. Rowland $\mathrm{M}$, Fleming $\mathrm{P}$, Bourke B. Looking in the mouth for Crohn's disease. Bowel Dis. 2010; 16: 332-337.

37. Tilakaratne WM, Freysdottir J, Fortune F. Orofacial granulomatosis: review on etiology and pathogenesis. J Oral Pathol Med 2008; 37: 191-195.

38. Kolho KL, Heiskanen K, Verkasalo M, Pitkäranta A. Orofacial granulomatosis in children- a challenge for diagnosis and treatment. Int J Pediatr Otorhinolaryngol 2011 Jun; 75(6): 864-7.

39. Wiesenfeld D, Ferguson MM, Mitchell DN et al. Oro-facial granulomatosis - a clinical and pathological analysis. Q J Med 1985; 54: 101-113.

40. Van der Waal RIF, Schulten EAJM, van der Meij EH et al. Cheilitis granulomatosa: overview of 13 patients with long-term follow-up - results of management. Int J Dermatol 2002; 41: 225-229.

41. Plauth M, Jenss H, Meyle J. Oral manifestations of Crohn's disease, an analysis of 79 cases. J Clin Gastroenterol 1991; 13: 29-37.

42. Bogenrieder T, Rogler G, Vogt T et al. Orofacial granulomatosis as the initial presentation of Crohn's disease in an adolescent. Dermatology 2003;206: 273-278.

43. Allen CM, Camisa C, Hamzeh S, Stephens L. Cheilitis granulomatosa: report of six cases and review of the literature. J Am Acad Dermatol 1990; 23: 444-50.

44. Friedrich W, Timmermann J Miescher's granulomatous cheilitis. Diagnostic and therapeutic aspects.Laryngorhinootologie 1990; 69: 564-8.

45. Alawi F. Granulomatous diseases of the oral tissues: differential diagnosis and update. Dent Clin North Am 2005; 49: 203-21.

46. Ayangco L, Rogers RS 3rd, Sheridan PJ. Pyostomatitis vegetans as an early sign of reactivation of Crohn's disease: a case report. J Periodontol 2002; 73: 1512-6.

47. Delaporte E, Viget N, Pasturel-Michon U, Catteau B, Hachulla E, Piette F. Pyostomatitispyodermatitis vegetans uncovering a case of Crohn disease. Ann Dermatol Venereol 1998;125: 331-4.

48. Hansen LS, Silverman S Jr, Daniels TE. The differential diagnosis of pyostomatitis vegetans and its relation to bowel disease. Oral Surg Oral Med Oral Pathol 1983; 55: 363-73. 
49. Greenstein AJ, Janowitz HD, Sachar DB. The extra-intestinal complications of Crohn's disease and ulcerative colitis: a study of 700 patients. Medicine (Baltimore) 1976; 55: 401-12.

50. Lisciandrano D, Ranzi T, Carrassi A et al. Prevalence of oral lesions in inflammatory bowel disease. Am J Gastroenterol 1996; 91: 7-10.

51. Field EA, Tyldesley WR. Oral Crohn's disease revisited-a 10-year review. Br J Oral Maxillofac Surg 1989; 27: 114-23.

52. Colella G, Riegler G, Lanza A, Tartaro GP, Russo MI, Tartaglione M. Changes in the mouth mucosa in patients with chronic inflammatory intestinal diseases. Minerva Stomatol 1999; 48: 367-71.

53. Lourenço SV, Hussein TP, Bologna SB, Sipahi AM, Nico MM. Oral manifestations of inflammatory bowel disease: a review based on the observation of six cases. J Eur Acad Dermatol Venereol 2010; 24: 204-7.

54. Correll RW, Wescott WB, Jensen JL. Recurring, painful oral ulcers. J Am Dent Assoc 1981; 103: 497-8.

55. Gargiulo AV, Ladone JA, Toto PD, Logiudice J. Crohn's disease: early detection by gingival biopsy. Periodontal Case Rep 1989; 11: 20-2.

56. Ciantar M, Adlam DM. Treatment with infliximab: implications in oral surgery? A case report. Br J Oral Maxillofac Surg 2007; 45: 507-10.

57. Roseth AG, Schmidt PN, Fagerhol MK. Correlation between faecal excretion of indium111-labelled granulocytes and calprotectin, a granylocyte marker protein, in patients with inflammatory bowel disease. Scand J Gastroenterol 1999; 34: 50-54.

58. Sipponen T, Savilahti E, Kolho KL, Nuutinen H, Turunen U, Fa“rlkkila“ M. Crohn's disease activity assessed by fecal calprotectin and lactoferrin: corrleation with Crohn's disease activity index and endoscopic findings. Inflamm Bowel Dis 2008; 14: 40-46.

59. Harikishan G, Reddy NR, Prasad H, Anitha S. Oral Crohn's disease without intestinal manifestations. J Pharm Bioallied Sci 2012;4:S431-4.

60. Peitsch WK, Kemmler N, Goerdt S, Goebler M. Infliximab: a novel treatment option for refractory orofacial granulomatosis. Acta Dermatol Venereol 2007; 87: 265-266.

61. Mantegazza C, Angiero F, Zuccotti GV. Oral manifestations of gastrointestinal diseases in children. Part 3: Ulcerative colitis and gastro-oesophageal reflux disease. Eur J Paediatr Dent 2016a;17(3):248-250.

62. Seo JK, Yeon KM, Chi JG. Inflammatory bowel disease in children--clinical, endoscopic, radiologic and histopathologic investigation. J Korean Med Sci 1992 Sep; 7(3):221-35.

63. Alstead EM, Wilson AG, Farthing MJ. Lichen planus and mesalazine. J Clin Gastroenterol 1991;13:335-37.

64. Calobrisi SD, Mutasim DF, McDonald JS. Pyostomatitis vegetans associated with ulcerative colitis. Temporary clearance with fluocinonide gel and complete remission after colectomy. Oral Surg Oral Med Oral Pathol Oral Radiol Endod 1995 Apr; 79(4):4524.

65. O'Loughlin S, Perry HO. A diffuse pustular eruption associated with ulcerative colitis. Arch Dermatol $1978 \mathrm{Jul}$; 114(7):1061-4.

66. Davies PT, Shadforth MF. Sulphasalazine induced oral lichen planus. Br Med J 1984;288:194.

67. Parvinen T, Parvinen I, Larmas $\mathrm{M}$. Stimulated salivary flow rate, $\mathrm{pH}$, and lactobacillus and yeast concentrations in medicated persons. Scand J Dent Res 1984;92:524-32.

68. Sreebny LM, Schwartz SS. A reference guide to drugs and dry mouth. Gerodontology 1986;5:75-99. 
69. Mijandrusi-Sinci B, Licul V, Gorup L, Brnci N, Glazar I, Lucin K. Pyostomatitis vegetans associated with inflammatory bowel disease-report of two cases. Coll Antropol 2010; 34 Suppl 2:279-82.

70. Folashade AJ, Melvin B. Heymant extraintestinal manifestations of inflammatory bowel disease. J Pediatr Gastroenterol Nutr 2008 Feb; 46(2): 124-133.

71. Lekovi Z, Radlovi N, Brdar R, Vuleti B, Jani N, Risti D, Stojsi Z, Radlovi V, Simi D, Nikoli D. Clinical characteristics of idiopathic ulcerative colitis in children. Srp Arh Celok Lek 2011 Mar-Apr;139(3-4):170-3.

72. Krebs KT. Case study: aphthous ulcers in a 14-year-old girl. Pediatr Nurs 2011 MayJun;37(3):115-8.

73. Kugathasan S, Judd RH, Hoffmann RG et al. Epidemiologic and clinical characteristics of children with newly diagnosed inflammatory bowel disease in Wisconsin: a statewide population-based study. J Pediatr 2003;143:525-31.

74. Bentsen BS, Moum B, Ekbom A. Incidence of inflammatory bowel disease in children in southeastern Norway: a prospective popula- tion-based study 1990 - 94. Scand J Gastroenterol 2002; 37:540-5.

75. Elahi M, Telkabadi M, Samadi V, Vakili H. Association of oral manifestations with ulcerative colitis. Gastroenterol Hepatol Bed Bench 2012 Summer; 5(3): 155-160.

76. Pace F, Pallotta S, Tonini M, Vakil N, Bianchi Porro G. Systematic review: gastrooesophageal reflux disease and dental lesions. Aliment Pharmacol Ther 2008 Jun;27(12):1179-86.

77. Vandenplas Y, Colin DR. Pediatric Gastroesophageal Reflux Clinical Practice Guidelines: Joint Recommendations of the North American Society for Pediatric Gastroenterology, Hepatology, and Nutrition (NASPGHAN) and the European Society for Pediatric Gastroenterology, Hepatology, and Nutrition (ESPGHAN). Committee Members: Di Lorenzo C, Hassall E, Liptak G, Mazur L, Sondheimer J, Staiano A, Thomson M, VeeremanWauters G, Wenzl TG. J Pediatric Gastroenterology Nutrition 2009; 49:498-547.

78. Pindborg JJ. Chemical and physical injuries. In: Pindborg JJ ed. Pathology of the dental hard tissues. Philadelphia: Saunders; 1970. p 312-325.

79. Dahshan A, Patel H, Delaney J, Wuerth A, Thomas R, Tolia V. Gastroesophageal reflux disease and dental erosion in children. J Pediatr. 2002 Apr;140(4):474-8.

80. Wilder-Smith $\mathrm{CH}$, Wilder-Smith $\mathrm{P}$, Kawakami-Wong $\mathrm{H}$, Voronets J, Osann K, Lussi A. Quantification of dental erosions in patients with GERD using optical coherence tomography before and after double-blind, randomized treatment with esomeprazole or placebo. Am J Gastroenterol 2009;104:2788-2795.

81. Di Fede O, Di Liberto C, Occhipinti G, Vigneri S, Lo Russo F, Fedele S, Lo Muzio S, Campisi G. Oral manifestations in patients with gastro-oesophageal reflux disease: a single-center case-control study. J Oral Pathol Med 2008;37: 336-340.

82. Silva MA, Damante JH, Stipp AC, Tolentino MM, Carlotto PR, Fleury RN. Gastroesophageal reflux disease: new oral findings. Oral Surg Oral Med Oral Pathol Oral Radiol Endod 2001; 91: 301-10.

83. Vakil N, van Zanten SV, Kahrilas P, Dent J, Jones R. Global Consensus Group. The Montreal definition and classification of gastroesophageal reflux disease: a global evidence-based consensus. Am J Gastroenterol 2006;101:1900-1920.

84. Barker JM, Liu Celiac Disease: Pathophysiology, clinical manifestations and associated autoimmune conditions. Adv Pediatr 2008; 55: 349-365.

85. Mantegazza C, Paglia M, Angiero F, Crippa R. Oral manifestations of gastrointestinal diseases in children. Part 4: Coeliac disease. Eur J Paediatr Dent 2016b;17(4):332-334. 
86. Lionetti E, Catassi C. Co-localization of gluten consumption and HLA-DQ2 and -DQ8 genotypes, a clue to the history of celiac disease. Dig Liver Dis 2014;46(12):1057e63.

87. Rashid M, Zarkadas M, Anca A, Limeback H. Oral manifestations of celiac disease: a clinical guide for dentists. J Can Dent Assoc 2011;77:b39

88. Tack GJ, Verbeek WHM, Schreurs MWJ, Mulder CJJ. The spectrum of celiac disease: epidemiology, clinical aspects and treatment. Nat Rev Gastroenterol Hepatol 2010; 7(4): 204-213.

89. Hill ID, Dirks MH, Liptak GS, Colletti RB, Fasano A, Guandalini S et al. Guideline for the diagnosis and treatment of celiac disease in children: recommendations of the North American Society for Pediatric Gastroenterology, Hepatology and Nutrition. J Pediatr Gastroenterol Nutr 2005;40(1):1-19.

90. Aine L, Mäki M, Collin P, Keyriläinen O. Dental enamel defects in celiac disease. J Oral Pathol Med 1990 Jul;19(6):241-5.

91. Campisi G, Di Liberto C, Carroccio A, Compilato D, lacono G, Procaccini M et al. Coeliac disease: oral ulcer prevalence, assessment of risk and association with gluten-free diet in children. Dig Liver Dis 2008 Feb;40(2):104-7. Epub 2007 Dec 11.

92. Condò $R$, Costacurta $M$, Maturo $P$, Docimo R. The dental age in the child with coeliac disease. Eur J Paediatr Dent 2011 Sep;12(3):184-8.

93. Sedghizadeh PP, Shuler CF, Allen CM, Beck FM, Kalmar JR. Celiac disease and recurrent aphthous stomatitis: a report and review of the literature. Oral Surg Oral Med Oral Pathol Oral Radiol Endod 2002 Oct;94(4):474-8.

94. Trandafir LM, Anton-Paduraru DT, Rusu D, Burlea M. Oral manifestations in celiac disease children. Romanian Journal of Oral Rehabilitation 2014 January - March; 6(1): 33-37.

95. Lähteenoja H, Toivanen A, Viander M, Mäki M, Irjala K, Räihä I et al. Oral mucosal changes in coeliac patients on a gluten-free diet. Eur J Oral Sci 1998; 106(5): 899-906.

96. Shetyer E, Berson T, Lachmanovitz O, Hidas A, Wilschanski M, Meneachem M, Shachar E, Shapira J, Steinberg D, Moskovitz M. Oral health status and salivary properties in relation to gluten free diet in children with celiac disease. J Pediatr Gastroenterol Nutr 2013, 57, 1, 49-52.

97. Benkebil F, Combescure C, Anghel SI, Besson Duvanel C, Schäppi MG. Diagnostic accuracy of a new point-of-care screening assayfor celiac disease. World J Gastroenterol 2013;19:5111-7.

98. Ertekin V, Selimoglu MA, Kardas F, Aktas, E. Prevalence of CD in Turkish children. J Clin Gastroenterol 2005; 39: 689-691.

99. Husby S, Koletzko S, Korponay-Szabó IR, Mearin ML, Phillips A,Shamir R et al. European Society for Pediatric Gastroenterology, Hepatology, and Nutrition guidelines for the diagnosis of coeliac disease. J Pediatr Gastroenterol Nutr 2012;54:136-60.

100. Mantegazza C, Crippa R, Zuccotti GV. Oral manifestations of gastrointestinal diseases in children. Part 1: General introduction. Eur J Paediatr Dent 2016a;17(1):80-82.

101. Mantegazza, C., et al. "An overview on oral manifestations of gastrointestinal diseases." Ital J Dent Med 5 (2020)

\section{DECLARAÇÃO CCBYNC}

Este artigo é uma cópia com adaptação para o português do original "Mantegazza, C., et al. "An overview on oral manifestations of gastrointestinal diseases." Ital J Dent Med 5 (2020): (101)

Esta cópia com adaptação para o português teve como alteração além do idioma, a autoria 
do artigo e lyout do PDF.

Os autores da publicação original não deram endosso específico a esta cópia com adaptação para a português, a não ser a licença CCBYNC 4.0 disponibilizada pelo periódico que publicou o artigo original.

Este é o link da licença: https://creativecommons.org/licenses/by/4.0/

Este é o link do artigo original: http://www.dentalmedjournal.it/an-overview-on-oralmanifestations-of-gastrointestinal-diseases/ 